\title{
Percolation and Phase Transitions in the Ising Model
}

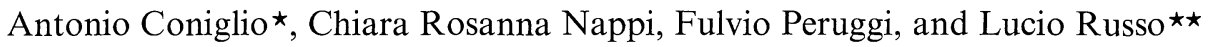 \\ Istituto di Fisica Teorica, Università di Napoli, I-80125 Napoli, Italy
}

\begin{abstract}
We give a description of the mechanism of phase transitions in the Ising model, pointing out the connection between the spontaneous magnetization and the existence of infinite clusters of "up" and "down" spins. The picture is more complete in the two-dimensional Ising model, where we can also use a generalized version of a result by Miyamoto.
\end{abstract}

\section{Introduction}

Percolation problems have been mostly studied for non-interacting systems (for a general review, see for example [1]). Only recently other cases have been considered: rigorous results are proved in [2], where site percolation problems for Ising spins on Bethe lattices are solved, and in [3], where Miyamoto extends to a class of interacting systems a classical result stated by Harris [4] for the random bond percolation problem on the plane square lattice.

In this paper we consider only site percolation problems, because in our picture they are more strictly related to the Ising model than the bond ones.

In Section 2 we consider the $v$-dimensional Ising model. We prove that, at zero external field and for $T<T_{c}$, percolation probability and spontaneous magnetization are related by an inequality.

We next limit ourselves to the case $v=2$. We first give a further extension of the theorem proved in [3] under the condition of "symmetry of configuration", observing that it has a natural generalization to the non-symmetric cases. Furthermore, we prefer to reformulate the statement for the site percolation problem using the matching graph of the plane square lattice rather than its dual graph. This is done in Section 3.

Finally, in Section 4 we simultaneously use the results of the preceding sections. We show that, at zero external field, in the single phase region there are no infinite clusters, while in the two phases region each pure phase is characterized by the existence of an infinite cluster of the corresponding sign.

* C.N.R., Gruppo Nazionale di Struttura della Materia

$\star \star$ C.N.R., Gruppo Nazionale di Fisica Matematica 


\section{Percolation and Spontaneous Magnetization}

In this section we obtain an inequality relating percolation probability and spontaneous magnetization in the $v$-dimensional Ising model. Before stating this result, we introduce some definition and notations.

$\boldsymbol{Z}^{v}$ is the $v$-dimensional lattice of the points with integral coordinates in $\boldsymbol{R}^{v}$.

We put $\Omega=\{-1,+1\}^{Z^{\nu}}$, and $\Omega_{\Lambda}=\{-1,+1\}^{\Lambda}$, for every finite set $\Lambda \subset \boldsymbol{Z}^{\nu}$. We consider in $\Omega$ the partial ordering $\leqq$ defined by setting $\omega_{1} \leqq \omega_{2}$ if and only if $\omega_{1}(x) \leqq \omega_{2}(x)$, for all $x \in \boldsymbol{Z}^{\nu}$. We also suppose that $\Omega$ is endowed with the topology obtained as product of the discrete topologies on the factors, and we call $\mathscr{B}$ the $\sigma$-algebra of Borel sets in $\Omega$.

Two points in $\boldsymbol{Z}^{\nu}$ which differ only by one unit in one coordinate are called adjacent points. A chain is a finite sequence $\left(x_{1}, \ldots, x_{n}\right)$ of distinct points in $\boldsymbol{Z}^{v}$ such that $x_{i-1}$ and $x_{i}$ are adjacent for every $i \in\{2, \ldots, n\}$. A subset $Y \subset \boldsymbol{Z}^{v}$ is connected if, for all pairs $x, y$ of points in $Y$, there is a chain formed by points in $Y$, having $x, y$ as terminal points. The boundary of $Y \subset Z^{v}$ is the set $\partial Y$ of all points in $Z^{\nu} \backslash Y$ that are adjacent to at least one point in $Y$.

If $\omega \in \Omega$, a (+)-cluster [(-)-cluster] in $\omega$ will be a maximal connected component of $\omega^{-1}(+1)\left[\omega^{-1}(-1)\right]$. We indicate infinite clusters by adding the symbol $\infty$ between the brackets.

Let $\mu$ be a probability measure defined on $\mathscr{B}$. Consider the events $C_{\infty}^{ \pm}=\{\omega \in \Omega \mid \mathbf{0}$ belongs to an $(\infty, \pm)$-cluster in $\omega\} ; E^{ \pm}=\{\omega \in \Omega \mid \omega(\mathbf{0})= \pm 1\}$; where $\mathbf{0}$ is the origin $(0, \ldots, 0) \in \boldsymbol{Z}^{v}$.

The percolation probability ${ }^{1}$ for "up" or "down" spins is defined by $R( \pm ; \mu)=$ $\mu\left(C_{\infty}^{ \pm}\right)$, and the "magnetization" is defined by $M(\mu)=\mu\left(E^{+}\right)-\mu\left(E^{-}\right)$.

We are interested in the ferromagnetic Ising model: in particular we shall consider the Gibbs measures $\mu_{+}$and $\mu_{-}$obtained by choosing respectively $(+)$-boundary conditions and $(-)$-boundary conditions.

Our main result is the following

Theorem 1. For a ferromagnetic Ising model at zero external field $(h=o)$ and below the critical temperature $\left(T<T_{c}\right)$ the following inequality holds: $\left|M\left(\mu_{ \pm}\right)\right| \leqq R\left( \pm ; \mu_{ \pm}\right)$.

Proof. Let $\Gamma$ be the family of all finite connected subset of $\boldsymbol{Z}^{v}$ containing the origin. For a fixed $Y \in \Gamma$ we consider a $v$-dimensional cube $\Lambda_{0}$ containing $Y \cup \partial Y$, and the set $\Lambda=\Lambda_{0} \cup \partial \Lambda_{0}$.

Let us denote by $\mu_{\Lambda}$ the Gibbs measure on $\Omega_{\Lambda}$ with zero boundary conditions, and by $C_{Y}^{+}\left[C_{Y}^{-}\right]$the set of configurations $\omega \in \Omega_{\Lambda}$ such that $Y$ is a $(+)$-cluster $[(-)$-cluster $]$ in $\omega$.

Finally, we set $B^{ \pm}=\left\{\omega \in \Omega_{\Lambda} \mid \omega(x)= \pm 1, \forall x \in \partial \Lambda_{0}\right\}$

Then, we have:

$$
\begin{aligned}
& \mu_{\Lambda_{0}}^{+}\left(C_{Y}^{+}\right) \equiv \mu_{\Lambda}\left(C_{Y}^{+} \mid B^{+}\right)=\frac{\mu_{\Lambda}\left(C_{Y}^{+}\right)}{\mu_{\Lambda}\left(B^{+}\right)} \mu_{\Lambda}\left(B^{+} \mid C_{Y}^{+}\right), \\
& \mu_{\Lambda_{0}}^{-}\left(C_{Y}^{+}\right) \equiv \mu_{\Lambda}\left(C_{Y}^{+} \mid B^{-}\right)=\frac{\mu_{\Lambda}\left(C_{Y}^{+}\right)}{\mu_{\Lambda}\left(B^{-}\right)} \mu_{\Lambda}\left(B^{-} \mid C_{Y}^{+}\right) .
\end{aligned}
$$

1 Note that our definition differs from the usual one, that is $P( \pm ; \mu)=\mu\left(C_{\infty}^{ \pm} \mid E^{ \pm}\right)=R( \pm ; \mu) / \mu\left(E^{ \pm}\right)$ 
Let us call $C_{\partial Y}^{-} \supset C_{Y}^{+}$the set of configurations $\omega \in \Omega_{\Lambda}$ such that $\omega(x)=-1$, for all $x \in \partial Y$. Since $\mu_{\Lambda}$ is invariant under interchange of -1 and +1 , and one-step Markov, we have:

$$
\frac{\mu_{\Lambda_{0}}^{+}\left(C_{Y}^{+}\right)}{\mu_{\Lambda_{0}}^{-}\left(C_{Y}^{+}\right)}=\frac{\mu_{\Lambda}\left(B^{+} \mid C_{Y}^{+}\right)}{\mu_{\Lambda}\left(B^{-} \mid C_{Y}^{+}\right)}=\frac{\mu_{\Lambda}\left(B^{+} \mid C_{\partial Y}^{-}\right)}{\mu_{\Lambda}\left(B^{-} \mid C_{\partial Y}^{-}\right)}=\frac{\mu_{\Lambda}\left(B^{+} \cap C_{\partial Y}^{-}\right)}{\mu_{\Lambda}\left(B^{-} \cap C_{\partial Y}^{-}\right)} .
$$

On the other hand, the FKG inequality [5] and the configurational symmetry of $\mu_{\Lambda}$ give:

$$
\mu_{\Lambda}\left(B^{+} \cap C_{\partial Y}^{-}\right) \leqq \mu_{\Lambda}\left(B^{+}\right) \mu_{\Lambda}\left(C_{\partial Y}^{-}\right)=\mu_{\Lambda}\left(B^{-}\right) \mu_{\Lambda}\left(C_{\partial Y}^{-}\right) \leqq \mu_{\Lambda}\left(B^{-} \cap C_{\partial Y}^{-}\right)
$$

where we have used the fact that the characteristic function $\chi_{B^{+}}$is increasing, while $\chi_{B^{-}}$and $\chi_{C_{\bar{\partial} Y}}$ are decreasing.

From (2.1) it follows that

$\mu_{\Lambda_{0}}^{+}\left(C_{Y}^{+}\right) \leqq \mu_{\Lambda_{0}}^{-}\left(C_{Y}^{+}\right)$.

This relation holds for all $\Lambda_{0} \supset Y \cup \partial Y$, so that in the limit $\Lambda_{0} \rightarrow \infty$, one obtain:

$$
\mu_{+}\left(C_{Y}^{+}\right) \leqq \mu_{-}\left(C_{Y}^{+}\right) \text {. }
$$

Now we also have:

$$
\mu_{+}\left(E^{+}\right)=\sum_{Y \in \Gamma} \mu_{+}\left(C_{Y}^{+}\right)+\mu_{+}\left(C_{\infty}^{+}\right) .
$$

This equality and the analogous one, obtained by interchange of + and - , give:

$$
\begin{aligned}
M\left(\mu_{+}\right) & =\mu_{+}\left(E^{+}\right)-\mu_{+}\left(E^{-}\right)=\mu_{+}\left(E^{+}\right)-\mu_{-}\left(E^{+}\right) \\
& =\sum_{Y \in \Gamma}\left[\mu_{+}\left(C_{Y}^{+}\right)-\mu_{-}\left(C_{Y}^{+}\right)\right]+\mu_{+}\left(C_{\infty}^{+}\right)-\mu_{-}\left(C_{\infty}^{+}\right) .
\end{aligned}
$$

Then, (2.2) and (2.3) imply $M\left(\mu_{+}\right) \leqq R\left(+; \mu_{+}\right)$.

Changing all signs, we can prove that $\left|M\left(\mu_{-}\right)\right| \leqq R\left(-_{-} ; \mu_{-}\right)$and the theorem follows.

\section{Non-Coexistence of Infinite Clusters in the Plane Square Lattice}

In this section we limit ourselves to the case $v=2$. Besides the definitions and notations of Section 2, we also need the following further definitions.

For every $K \subset \boldsymbol{Z}^{2}$, we call $\mathscr{B}_{K} \subset \mathscr{B}$ the $\sigma$-algebra generated by the functions $\omega \rightarrow \omega(x), x \in K$. We put $\mathscr{B}_{\infty}=\cap_{K} \mathscr{B}_{K^{c}}$, where $K$ runs over the class of all finite subsets of $\boldsymbol{Z}^{2}$.

A circuit in $Z^{2}$ is a chain $\left(x_{1}, \ldots, x_{n}\right)$ such that $x_{i}$ and $x_{j}$ are adjacent only if $|i-j|$ is 1 or $n-1$.

Two points in $\boldsymbol{Z}^{2}$ that are adjacent or such that both their coordinates differ by one unit are called $(*)$-adjacent points. We define $(*)$-chains, $(*)$-circuits, (*)-connection, $(*)$-boundary, and $( \pm, *)$-clusters in the same way as chains, circuits, connection, boundary, and $( \pm)$-clusters, only replacing adjacency with (*)-adjacency. 
Note that every connected [(*)-connected] finite subset of $\boldsymbol{Z}^{2}$ is "surrounded" by a $(*)$-circuit [circuit] contained in its boundary $[(*)$-boundary].

Let us call $\boldsymbol{E}$ and $\boldsymbol{E}^{*}$ the sets of segments in $\boldsymbol{R}^{2}$ which we obtain connecting all pairs of points in $\boldsymbol{Z}^{2}$ that are respectively adjacent and (*)-adjacent. In the language of graph theory, the graph $\left(\boldsymbol{Z}^{2}, \boldsymbol{E}^{*}\right)$ is the matching graph of the simple planar graph $\left(\boldsymbol{Z}^{2}, \boldsymbol{E}\right)$.

Now we can prove the following:

Theorem 2. If $\mu$ is a translationally invariant equilibrium measure for a ferromagnetic two-dimensional Ising model at zero external field and $\mu$ is extremal in the set of all equilibrium measures, then:

$$
R(-; \mu) R(+; \mu)=0 .
$$

First we observe that the hypotheses of the theorem are equivalent to the following set of conditions (see $[5,6,7]$ ):

a) spatial symmetry: $\mu$ is invariant under translations, rotations by right angles and reflections in the axes,

b) $\mu$ is everywhere dense,

c) $\mathscr{B}_{\infty}$ is trivial if it is measured by $\mu$,

d) the FKG inequality holds for $\mu$,

e) $\mu$ is one-step Markov,

f) configurational symmetry of conditional probabilities: if $C$ is a cylinder with base $A$ and $B$ is a boundary condition on a (*)-circuit surrounding $A, \mu(C \mid B)=$ $\mu\left(C^{\sim} \mid B^{\sim}\right)$, where tilde means interchange of + and - .

The theorem can be proved following in the essential lines the procedure of Ref. [3] and [4]. The main changes with respect to Miyamoto's proof are due to the weakening of configurational symmetry (which allows us to extend the theorem to the region $T<T_{c}$ ) and to an oversight that is contained in [3] in the proof of Lemma 4 (namely an incorrect use of the Markov property). Furthermore, where (as in Lemmata 1 and 2) the proof is essentially the same as in [3] the site terminology (necessary in order to give a description of the usual Ising model in terms of an one-step two dimensional Markov process) allows us to simplify the technical details. So, for the convenience of the reader, we give below the complete proof of the theorem.

It is known $([9,10])$ that the measures satisfying the hypotheses of Theorem 2 are at most two, namely the measures $\mu_{+}$and $\mu_{-}$. (These measures in the region $T \geqq T_{c}$ coincide); we prove the theorem in the case $\mu=\mu_{+}$; in the case $\mu=\mu_{-}$the proof is obviously the same.

Before proving the theorem we need some lemmata.

Lemma 1. Call $R\left(\pi / 2, \pm ; \mu_{+}\right)$the $\mu_{+}$-measure of the event that $\mathbf{0}$ belongs to an infinite connected component of $\omega^{-1}( \pm 1) \cap\{x \geqq 0 ; y \geqq 0\}$.

Then we have:

$$
R\left(\pi / 2,-; \mu_{+}\right)=0 \text {. }
$$

Proof. It is easy to check that $R\left(\pi / 2,-; \mu_{+}\right) \leqq R\left(\pi / 2,+; \mu_{+}\right)$so that it suffices to demonstrate that $R\left(\pi / 2,+; \mu_{+}\right)>0$ implies $R\left(\pi / 2,-; \mu_{+}\right)=0$. 
For a positive integer $j$, let $E_{j}$ be the event that the point $(0, j)$ belongs to an infinite connected component of $\omega^{-1}(+1) \cap\{x \geqq 0 ; y \leqq j\}$.

Condition (a) implies $\mu_{+}\left(E_{j}\right)=R\left(\pi / 2,+; \mu_{+}\right)$.

Then, by condition (c), we can use Birkhoff's ergodic theorem, which assures that frequency of occurrence of $E_{1}, E_{2}, \ldots$ is equal to $R\left(\pi / 2,+; \mu_{+}\right) \mu_{+}$-a.e.

Therefore, infinitely many $E_{j}$ 's occur $\mu_{+}$-a.e.

Assume that the event $E_{j}$ occurs. For a positive integer $k$ let $B_{k}$ be the event that $\omega(v)=-1$ for each point $v$ on the line $\{x=k, 0 \leqq y \leqq j\}$. Since $\mu_{+}\left(B_{k}\right)>0$ by (b), Birkoff's ergodic theorem implies that infinitely many $B_{k}$ 's occur $\mu_{+}$-a.e., so that the infinite connected component appearing in the event $E_{j}$ crosses the $x$-axis $\mu_{+}$-a.e. Thus, we have $\mu_{+}$-a.e. infinitely many $(+)$-chains connecting points on the $x$-axis to points on the $y$-axis, each of which blocks any $(-)$-chain in $\{x \geqq 0, y \geqq 0\}$ starting from the origin. This proves that $R\left(\pi / 2,-; \mu_{+}\right)=0$.

Remark. For any point $v$ in $\{x \geqq 0, y \geqq 0\}$ the event $X_{v}^{ \pm}$that $v$ belongs to an infinite connected component of $\omega^{-1}( \pm 1) \cap\{x \geqq 0, y \geqq 0\}$ has $\mu_{+}$-measure zero if and only if $R\left(\pi / 2, \pm ; \mu_{+}\right)=0$. Indeed, if $Q_{ \pm}$is the event that $v$ and $\mathbf{0}$ are connected in $\omega^{-1}( \pm 1) \cap\{x \geqq 0 ; y \geqq 0\}$, using (b) and (d) we have:

$$
\begin{aligned}
R\left(\pi / 2, \pm ; \mu_{+}\right) & =\mu_{+}\left(X_{0}^{ \pm}\right) \leqq \mu_{+}\left(X_{0}^{ \pm} \cap Q_{ \pm}\right) / \mu_{+}\left(Q_{ \pm}\right) \leqq \mu_{+}\left(X_{v}^{ \pm}\right) / \mu_{+}\left(Q_{ \pm}\right) \\
& \leqq \mu_{+}\left(X_{v}^{ \pm} \cap Q_{ \pm}\right) / \mu_{+}\left(Q_{ \pm}\right)^{2} \leqq \mu_{+}\left(X_{0}^{ \pm}\right) / \mu_{+}\left(Q_{ \pm}\right)^{2} .
\end{aligned}
$$

We shall omit an analogous remark after Lemma 2.

Lemma 2. If we call $R\left(\pi, \pm ; \mu_{+}\right)$the $\mu_{+}$-measure of the event that $\mathbf{0}$ belongs to an infinite connected component of $\omega^{-1}( \pm 1) \cap\{y \geqq 0\}$, we have:

$$
R\left(\pi,-; \mu_{+}\right)=0 \text {. }
$$

Proof. Let us suppose $R\left(\pi,-; \mu_{+}\right)>0$. For a positive integer $j$, let $W_{j}$ be the event that the point $(j, 0)$ belongs to an infinite connected component of $\omega^{-1}(-1) \cap\{y \geqq 0\}$ and to an infinite connected component of $\omega^{-1}(-1) \cap\{y \leqq 0\}$. By properties (a) and $(\mathrm{d})$, we have $\mu_{+}\left(W_{j}\right) \geqq R^{2}\left(\pi,-; \mu_{+}\right)>0$. Then, Birkoff's ergodic theorem implies that infinitely many $W_{j}$ 's occur $\mu_{+}$-a.e. Assume that the event $W_{j}$ occurs. Lemma 1 and the remark prove that the infinite connected components which appear in $W_{j}$ cross $\mu_{+}$-a.e. the $y$-axis respectively above and below the origin. Hence, we have, $\mu_{+}$-a.e., infinitely many $(-)$-chains connecting points above and below the $y$-axis, each of that blocks any $(+)$-chain in $\{y \geqq 0\}$ starting from the origin. This proves that $R\left(\pi,+; \mu_{+}\right)=0$. On the other hand we have $R\left(\pi,+; \mu_{+}\right) \geqq R\left(\pi,-; \mu_{+}\right)$, so that $R\left(\pi,-; \mu_{+}\right)=0$ and this concludes the proof.

$\mathrm{A}( \pm, *)$-chain which starts at a point on the $y$-axis, ends at a point on the $y$-axis below the starting point, and all points of which are in $\{x \geqq 0\}$ is called $( \pm)$-half-circuit. We call box a square with its centre in the origin.

Lemma 3. There exists an increasing sequence $\left\{V_{n}\right\}_{n=1}^{\infty}$ of boxes such that for all $n$ in $\left(V_{n+1} \backslash V_{n}\right) \cap\{x \geqq 0\}$ there is with $\mu_{+}$-measure $>2^{-2} a(+)$-half-circuit $(*)$-connected in $\omega^{-1}(1) \cap\left(V_{n+1} \backslash V_{n}\right) \cap\{x \geqq 0\}$ with a side of $V_{n+1}$ and, with $\mu_{+}$-measure $>2^{-2}, a(+)$-half-circuit $(*)$-connected in the same set with a side of $V_{n}$.

Proof. First, we prove that the event that for any box $V$ there exists a $(+)$-halfcircuit surrounding the origin and lying in $\{x \geqq 0\} \backslash V$ has $\mu_{+}$-measure 1 . 
Since $R\left(\pi,-; \mu_{+}\right)=0$, there is $\mu_{+}$-a.e. at least one $(+)$-half-circuit surrounding each point of the $y$-axis. The union of all the $(+)$-circuits surrounding $(0, j)$ can be divided into connected components $C_{1}^{j}, C_{2}^{j}, \ldots$ For the measure $\mu_{+}$conditioned to the event that these components are infinitely many, the statement above is obviously true; so we can suppose that the components $C_{1}^{j}, C_{2}^{j}, \ldots$ are finitely many. Then, there exists a maximal component $C_{M}^{j}$.

If $C_{M}^{j} \cap C_{M}^{j^{\prime}}=\emptyset$ for $j \neq j^{\prime}$, there is a $(-, \infty)$-cluster in $\{x \geqq 0\}$ starting from a point between $(0, j)$ and $\left(0, j^{\prime}\right)$, and this is absurd by property (a) and Lemma 2 . Therefore, for all $j$, there exists a common maximal component $C_{M} . \partial C_{M} \cap\{x \geqq 0\}$ can contain finite clusters only, so that it is easy to realize that in $C_{M}$ there are infinitely many (+)-half-circuits lying in $\{x \geqq 0\} \backslash V$. Choosing arbitrarily a box $V_{1}$, we can construct a sequence $\left\{V_{l}\right\}_{l=1}^{\infty}$ of boxes such that, for all $l$, with probability $>1 / 2$ there exists a $(+)$-half-circuit surrounding the origin and lying in $\{x \geqq 0\} \cap\left(V_{l+1} \backslash V_{l}\right)$. For a fixed $l$, if none of the above-mentioned (+)-half-circuits is $(*)$-connected in $\omega^{-1}(1)$ with a side of $V_{l+1}\left[V_{l}\right]$, there exists a (-)-half-circuit surrounding them [surrounded by them] lying in $\{x \geqq 0\} \cap\left(V_{l+1} \backslash V_{l}\right)$, and (*)-connected in $\omega^{-1}(1)$ with a side of $V_{l+1}\left[V_{l}\right]$.

Using correlation inequalities ([8]), it is easy to see that the Lemma holds.

Lemma 4. There exists an increasing sequence $\left\{B_{n}\right\}_{n=1}^{\infty}$ of boxes such that, for all $n$, in $B_{n+1} \backslash B_{n}$ there is a $(*,+)$-circuit surrounding the origin with $\mu_{+}$-probability $p>2^{-30}\left[R\left(+; \mu_{+}\right)\right]^{4}$.

Proof. We put $B_{n}=V_{3 n}$, where the $V_{n}$ are the boxes of Lemma 3; for each $n$ we choose an integer $i_{n}$ such that the point $\left(0, i_{n}\right)$ is in $V_{3 n+2} \backslash V_{3 n+1} \subset B_{n+1} \backslash B_{n}$.

Let $C^{ \pm}$be the event that in $B_{n+1} \backslash B_{n}$ there is a $(*, \pm)$-circuit surrounding the origin.

If $S[s]$ is a half-circuit in $B_{n+1} \backslash V_{3 n+2}\left[V_{3 n+1} \backslash B_{n}\right]$, we call $E(S)[E(s)]$ the event that $S[s]$ is the maximal [minimal] (+)-half-circuit in $B_{n+1} \backslash V_{3 n+2}\left[V_{3 n+1} \backslash B_{n}\right]$ and that it is $(*)$-connected in $\omega^{-1}(1) \cap\{x \geqq 0\}$ with a side of $B_{n+1}\left[B_{n}\right]$.

Define also:

$$
S_{r}=S \cap\{x>0\} ; \quad s_{r}=s \cap\{x>0\} ; \quad S_{0}=S \cap\{x=0\} ; \quad s_{0}=s \cap\{x=0\} .
$$

Let $S_{l}\left[s_{l}\right]$ be the reflection of $S_{r}\left[s_{r}\right]$ in the $y$-axis, $S_{t}=S \cup S_{l}, s_{t}=s \cup s_{l}$ and $S_{u}\left[s_{u}\right]$ the union of $S_{t}\left[s_{t}\right]$ and its interior. Let $D(S)$ be the event that $\left(0, i_{n}\right)$ is $(*)$-connected in $\omega^{-1}(1) \cap\left(S_{u} \backslash B_{n}\right)$ with $S$ and $D(s)$ the event that $\left(0, i_{n}\right)$ is $(*)$-connected in $\omega^{-1}(1) \cap$ $\left(\left(B_{n+1} \backslash s_{u}\right) \cup s_{t}\right)$ with $s$. Further we put $D^{M}=\cup_{S}(E(S) \cap D(S)) ; D^{m}=\cup_{s}(E(s) \cap D(s))$. We note that the sets in the unions are pairwise disjoint and $D^{M}, D^{m}$ are positive events (i.e. their characteristic functions are increasing). Finally we define $A=D^{M} \cup D^{m} \cup C^{+}$.

$A$ is obviously a positive event too.

We have

$$
\mu_{+}(A) \geqq \sum_{S, s} \mu_{+}\left(A \cap E_{S s}\right)=\sum_{S, s} \mu_{+}\left(A \mid E_{S s}\right) \mu_{+}\left(E_{S s}\right)
$$

where

$$
E_{S s}=E(S) \cap E(s) .
$$


If we call $C_{S s}^{ \pm}$the event that in $\left(S_{u} \backslash S_{u}\right) \cup s_{t}$ there is a $(*, \pm)$-circuit surrounding the origin and $E_{S s}^{\alpha}$ the intersection of $E_{S s}$ with the event that a given configuration $\alpha$ realizes on $S_{l} \cup S_{l}$, and if $E_{S s}^{\prime}$ is the event obtained by choosing $\alpha$ such that $\omega(v)=-1$ for all $v \in S_{l} \cup s_{l}$, by FKG inequality $([5,8])$ we have:

$$
\begin{aligned}
\mu_{+}\left(A \mid E_{S s}\right) & =\mu_{+}\left(E_{S s}\right)^{-1} \sum_{\alpha} \mu_{+}\left(A \mid E_{S s}^{\alpha}\right) \mu_{+}\left(E_{S s}^{\alpha}\right) \\
& \geqq \mu_{+}\left(A \mid E_{S s}^{\prime}\right) \geqq \mu_{+}\left(A_{S s} \mid E_{S s}^{\prime}\right)=\mu_{+}\left(A_{S s} \mid E_{S s}^{*}\right)
\end{aligned}
$$

where

$$
A_{S s}=D(S) \cup D(s) \cup C_{S s}^{+}
$$

and $E_{S s}^{*}$ is the event obtained by dropping in $E_{S s}^{\prime}$ the maximality and minimality conditions. The last equality holds by the Markov property. We call $F_{S s}^{+}\left[F_{S s}^{-}\right]$ the event that $i_{n}$ is surrounded in $\left(S_{u} \backslash S_{u}\right) \cup s_{t}$ by a $(+, *)$-circuit $[(-, *)$-circuit $]$ (which may be also coincident with $i_{n}$ ), not surrounding the origin, which is (*)-connected in $\omega^{-1}(1)\left[\omega^{-1}(-1)\right]$ with $S \cup s\left[S_{l} \cup s_{l}\right]$.

It is easy to check that $E_{S s}^{*}=E_{S s}^{+} \cup E_{S s}^{-}$where

$$
E_{S s}^{+}=\left(F_{S s}^{+} \cup C_{S s}^{+}\right) \cap E_{S s}^{*} ; \quad E_{S s}^{-}=\left(F_{S s}^{-} \cup C_{S s}^{-}\right) \cap E_{S s}^{*} .
$$

Let us define

$$
E_{S s}^{0}=\left\{\omega \in \Omega \mid \omega(x)=1, \forall x \in S_{r} \cup S_{r} ; \omega(x)=-1, \forall x \in S_{0} \cup S_{e} \cup S_{0} \cup S_{l}\right\} .
$$

Then it easily follows

$$
\begin{aligned}
\mu_{+}\left(E_{S s}^{+}\right) & =\mu_{+}\left(F_{S s}^{+} \cup C_{S s}^{+} \mid E_{S s}^{*}\right) \mu_{+}\left(E_{S s}^{*}\right) \geqq \mu_{+}\left(F_{S s}^{+} \cup C_{S s}^{+} \mid E_{S s}^{0}\right) \mu_{+}\left(E_{S s}^{*}\right) \\
& =\mu_{+}\left(F_{S s}^{-} \cup C_{S s}^{-} \mid E_{S s}^{*}\right) \mu_{+}\left(E_{S s}^{*}\right)=\mu_{+}\left(E_{S s}^{-}\right)
\end{aligned}
$$

by using properties (a) and (f), and the relation ([8]):

$$
\mu_{1}(A \cup B) \mu_{2}(A \cap B) \geqq \mu_{1}(A) \mu_{2}(B) .
$$

This inequality is satisfied by the measures $\mu_{1}(\cdot) \equiv \mu_{+}\left(\cdot \mid E_{S s}^{*}\right)$ and $\mu_{2}(\cdot) \equiv \mu_{+}\left(\cdot \mid E_{S s}^{0}\right)$ because the conditioning w.r.t. the event $E_{S s}^{*}$ introduces an external field larger than the one introduced by the conditioning w.r.t. the event $E_{S s}^{0}$.

Hence

$$
\mu_{+}\left(A_{S s} \mid E_{S s}^{*}\right) \geqq \frac{1}{2} \mu_{+}\left(A_{S s} \mid E_{S s}^{+}\right) .
$$

On the other hand we have

$$
\begin{aligned}
\mu_{+}\left(A_{S s} \mid E_{S s}^{+}\right) & =\mu_{+}\left(D(S) \cup D(s) \cup C_{S s}^{+} \mid E_{S s}^{*} \cap\left(F_{S s}^{+} \cup C_{S s}^{+}\right)\right) \\
& =\mu_{+}\left(D(S) \cup D(s) \cup\left(C_{S s}^{+} \cap E_{S s}^{*}\right) \mid\left(E_{S s}^{*} \cap F_{S s}^{+}\right) \cup\left(C_{S s}^{+} \cap E_{S s}^{*}\right)\right) \\
& \geqq \mu_{+}\left(D(S) \cup D(s) \mid E_{S s}^{*} \cap F_{S s}^{+}\right) .
\end{aligned}
$$

Applying again the Markov property and FKG inequality we get

$$
\mu_{+}\left(A_{S s} \mid E_{S s}^{+}\right) \geqq \mu_{+}(H) \geqq R\left(+; \mu_{+}\right)
$$


where $H$ is the event that $i_{n}$ belongs to a $(+, *, \infty)$-cluster. Collecting together (3.1), (3.2), (3.3), (3.4), we have:

$$
\mu_{+}(A) \geqq \sum_{S, s} \mu_{+}\left(E_{S S}\right) \frac{1}{2} R\left(+; \mu_{+}\right) \geqq 2^{-5} R\left(+; \mu_{+}\right)
$$

where, in the last inequality, we have used Lemma 3, the FKG inequality and the remark that $\cup_{S} E_{S}$ and $\cup_{s} E_{s}$ are positive events.

(3.5) and the definition of $A$ imply that, either

$$
\mu_{+}\left(C^{+}\right) \geqq 2^{-6} R\left(+; \mu_{+}\right)
$$

(and in this case the lemma is proven) or

$$
\mu_{+}\left(D^{M} \cup D^{m}\right) \geqq 2^{-6} R\left(+; \mu_{+}\right) .
$$

If (3.6) holds we can suppose that

$$
\mu_{+}\left(D^{M}\right)>2^{-7} R\left(+; \mu_{+}\right)
$$

(otherwise an analogous inequality holds for $D^{m}$ and the following part of the proof should be the same).

If (3.7) holds, by spatial symmetry, the same inequality holds for the event $D^{\prime}{ }^{M}$, obtained from $D^{M}$ by interchanging the point $\left(0, i_{n}\right)$ with the point $\left(0,-i_{n}\right)$, so that, by $\mathrm{FKG}$ inequality, we get

$$
\mu_{+}\left(D^{M} \cap D^{\prime M}\right) \geqq 2^{-14}\left[R\left(+; \mu_{+}\right)\right]^{2} .
$$

If the event $D^{M} \cap D^{\prime M}$ occurs, there exists a $(+, *)$ chain connecting $\left(0, i_{n}\right)$ and $\left(0,-i_{n}\right)$ in $B_{n+1} \backslash B_{n}$; by spatial symmetry and (3.8) such a chain exists and is clockwise with a probability bigger than $2^{-15}\left[R\left(+; \mu_{+}\right)\right]^{2}$.

Finally with a probability bigger than $2^{-30}\left[R\left(+; \mu_{+}\right)\right]^{4}$ both clockwise and anti-clockwise $(+, *)$ chains exist connecting $\left(0, i_{n}\right)$ and $\left(0,-i_{n}\right)$, so that the required $(*)$-circuit exists.

Proof of the Theorem 2. If $R\left(+, \mu_{+}\right)>0$, Lemma 4 holds with a positive value of $p$ and, using the $K$-mixing property of $\mu_{+}$, one can prove that a $(+, *)$-circuit surrounding the origin exists $\mu_{+}$-a.e.; hence $R\left(-, \mu_{+}\right)=0$. For details see Ref. [3].

\section{Percolation in the Two-dimensional Ising Model}

In this section we examine the consequence of Theorems 1 and 2 in the twodimensional Ising model.

Proposition 1. In a ferromagnetic Ising model at zero external field for $T \geqq T_{c}$ there are no infinite clusters, while for $T<T_{c}$ in each pure phase there is a.e. an infinite cluster of the corresponding sign and no infinite clusters of the opposite sign.

Proof. For $T \geqq T_{c} \mu_{+}=\mu_{-}=\mu$, so that Theorem 2 implies

$$
0=R(-, \mu) R(+, \mu)=R(-, \mu)^{2}=R(+, \mu)^{2}
$$

2 We are indebted to D. Ruelle for having suggested these points 
and this proves the first part of the proposition; the second part is an immediate consequence of Theorems 1 and 2.

In the case $h \neq 0$ we can only state that:

Proposition 2. If $\mu_{h}$ is the translationally invariant equilibrium measure for a ferromagnetic Ising model at external field $h \neq 0$

$$
R\left(-, \mu_{h}\right) R\left(+, \mu_{h}\right)=0 ;
$$

for $T<T_{c}$ there is $\mu_{h}$-a.e. an infinite cluster of the same sign as the external field and no infinite clusters of opposite sign.

Proof. It is an obvious consequence of Proposition 1 and of the remark that $R\left(+, \mu_{h}\right)$ is an increasing function of $h$ (as easily follows from the inequalities stated in $[8])^{2}$.

We remark that the rotation invariance is not necessary in proving Theorem 2 , so that the theorem can be extended to the anysotropic Ising model ${ }^{2}$.

In this case the only change in the proof should be that one must define, instead of $R(\pi, \pm ; \mu)$, the four quantities $R_{x}(\pi, \pm ; \mu)$ and $R_{y}(\pi, \pm ; \mu)$ (with an obvious meaning of the symbols) and Lemma 2 should assume the form $R_{x}\left(\pi,-; \mu_{+}\right) R_{y}\left(\pi,-; \mu_{+}\right)=0$; however, once one has chosen the axis corresponding to a null $R$ the other proofs remain unaltered.

Finally, we note that, with some technical changes, Theorem 2 can be easily extended to "sufficiently" regular planar graphs.

Acknowledgement. We would like to thank Prof. G. Gallavotti for having drawn our attention on Miyamoto's paper and for many helpful discussions, and Prof. D. Ruelle for helpful comments.

\section{References}

1. Essam,J.W.: Percolation and cluster size. In: Phase Transitions and Critical Phenomena (C.Domb and M.S. Green, ed.), Vol. II. New York: Academic Press 1972

2. Coniglio, A.: Phys. Rev. B13, 2194-2207 (1976)

3. Miyamoto, M.: Commun. math. Phys. 44, 169-173 (1975)

4. Harris, T.E.: Proc. Camb. Phil. Soc. 43, 13-20 (1960)

5. Fortuin, C.M., Kasteleyn, P. W., Ginibre, J.: Commun. math. Phys. 22, 89-103 (1971)

6. Lanford III, O.E., Ruelle, D.: Commun. math. Phys. 13, 194-215 (1969)

7. Spitzer, F.: Am. Math. Monthly 78, 142-154 (1971)

8. Holley, R.: Commun. math. Phys. 36, 227-231 (1974)

9. Lebowitz, J., Martin-Löf, A.: Commun. math. Phys. 25, 276-282 (1972)

10. Messager, A., Miracle-Sole, S.: Commun. math. Phys. 40, 187-196 (1975)

Communicated by G. Gallavotti

Received January 9, 1976; in revised form May 19, 1976 
